\title{
Randomised Controlled Trial Of Low (4-5mL/Kg) Vs. High (7-8 mL/Kg) Tidal Volume Using Volume Guarantee Ventilation - A Pilot Study
}

\section{A. Gupta', S. Gupta', C. Harikumar', S. Donn².}

1. North Tees and Hartlepool Foundation Trust, Paediatrics and Neonates, Stockton-on-Tees, United Kingdom. 2. University of Michigan, Pediatrics, Ann Arbor, USA.

Introduction

- Very Low Birth Weight Infants (<1500 grams) are born with surfactant deficiency and many require invasive mechanical support.

- Volume targeting appears to be a better modality than pressure targeting.

- Since volume guarantee (VG) helps to provide a more precise tidal volume, the debate has now shifted to what the ideal tidal volume should be.

- The recommended tidal volume varies from 4-8 $\mathrm{mL} / \mathrm{kg}$, but the optimal tidal volume has yet to be determined.

- To date, no study has addressed a comparison of "low" (4-5 mL/kg) versus "high" tidal volume (7-8 $\mathrm{mL} / \mathrm{kg})$ in premature infants with respiratory distress syndrome (RDS).

\section{Aims and Objectives}

To compare a lower (4-5 mL/kg) to a higher $(7-8 \mathrm{~mL} / \mathrm{kg})$ tidal volume during volume guarantee ventilation of preterm infants with RDS

\section{Methodology}

- This randomised trial was conducted at North Tees Hospital from 2013-2016.

- Inclusion criteria:

- Preterm newborns $<32$ weeks'gestation or $<1500$ grams birthweight with RDS, who required mechanical ventilation within 12 hours of birth

- Exclusion criteria

- Serious congenital anomalies

- Congenital diaphragmatic hernia

- Cyanotic congenital heart disease

- Airway anomalies

Abdominal wall defects

prior to transfer from other centres

- Subjects were randomised to receive lower $(4-5 \mathrm{~mL} / \mathrm{kg})$ or higher $(7-8 \mathrm{~mL} / \mathrm{kg})$ tidal volume using VG. The dead space was standardized by using prescribed trimming of the external portion of the endotracheal tube.

- All subjects received surfactant and were managed by a strict protocol (Figure 1) with allowed rescue by high frequency ventilation for defined criteria.

- The primary outcome was the time to achieve a $25 \%$ reduction from the initial peak inspiratory pressure (PIP).

- Secondary outcomes included the duration of mechanical ventilation, and common respiratory and non-respiratory complications.

- Time-sensitive variables were analysed using survival statistics (Kaplan-Meier and log rank test). Categorical variables were analysed by Chi-square and continuous variables by independent t-test; non-parametric parameters were assessed by the Wilcoxon rank sum test. Linear analysis was performed by Cox regression, and significant variables underwent multivariate analysis. The analyses were performed using SPSS $\circledast$ version 20.0 .

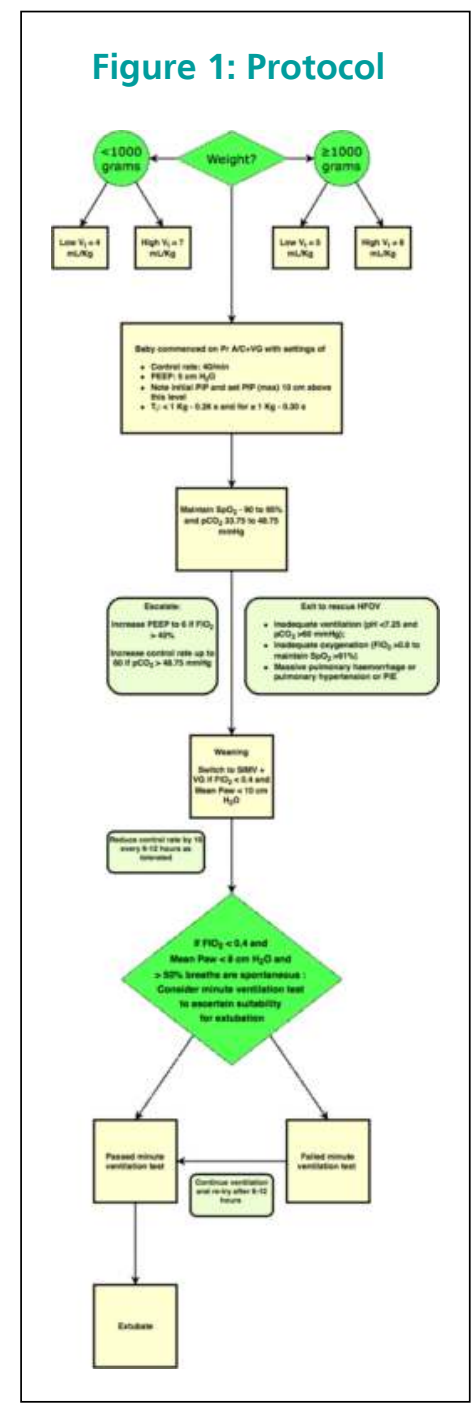

\section{Results}

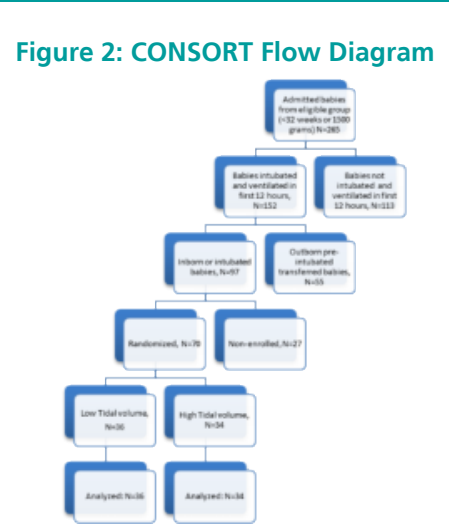

Table 1: Demographic Data and Disease Severity at Baseline

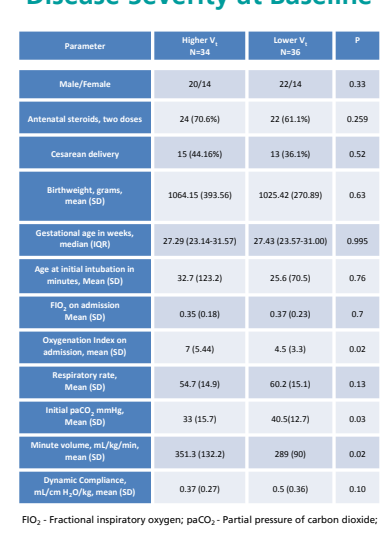

There were 97 eligible infants; 70 were randomised (Figure 2)

- High and Low groups were similar in their demographic profiles (Table 1)

- The primary outcome, time to reduce PIP by $25 \%$ (median [IQR]), was 13.6 [8.8 - 25.2] hours and 17.4 [7.7 $27.8]$ hours, respectively, for higher and lower tidal volumes $(p=0.678)$. (Table 2, Figure 3 )

- The total duration of mechanical ventilation (median [IQR]) for higher vs lower tidal volume was 33.3 (22 $368.8)$ and $61.8(15.4-177.5)$ hours, respectively $(p=0.959)$. (Table 2, Figure 4)

- Though tidal volume and PIP were different between groups, minute volume and $\mathrm{pCO}_{2}$ were not. (Table 3 Figure 5)

- There were no differences between the groups for respiratory and non-respiratory complications. (Table 2, Figure 6)
Primary and Secondary Outcomes

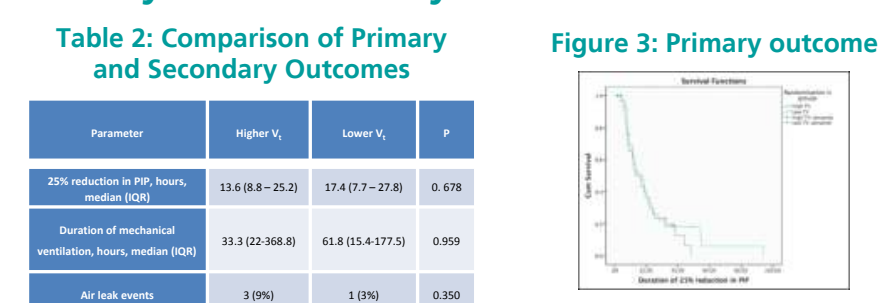

Figure 4: Total duration of mechanical ventilation
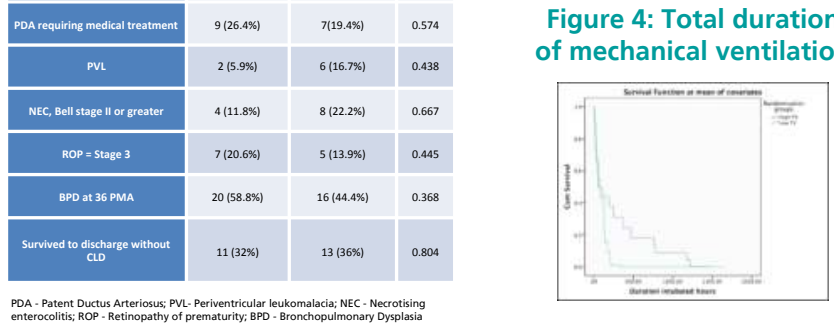

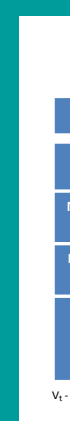

Table 3: Ventilatory parameters

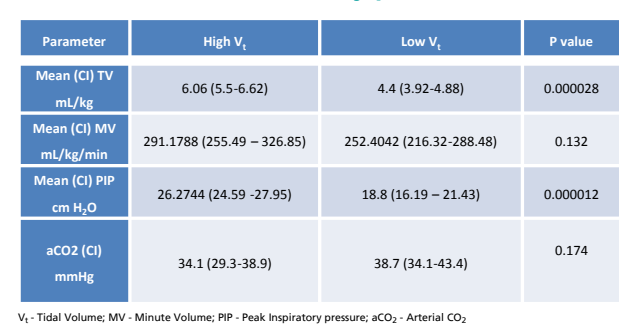

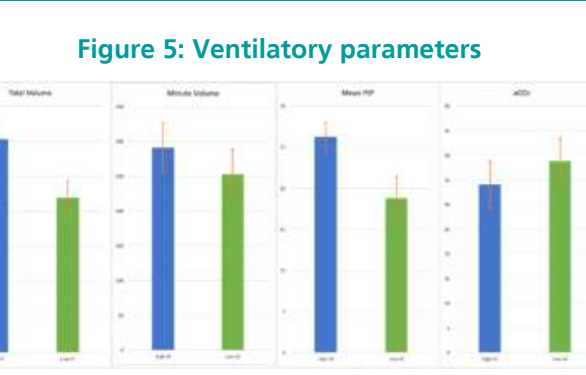

Figure 6: Pulmonary and non-pulmonary complications

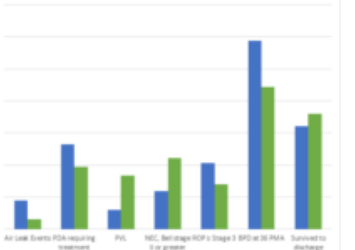

Summary and Conclusions

Figure 7

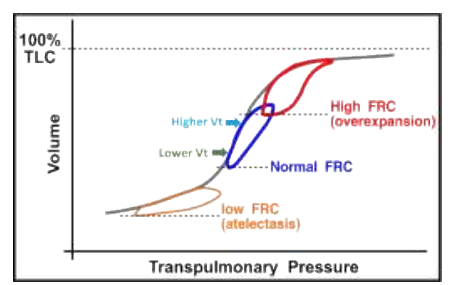

- This small pilot trial failed to demonstrate differences attributable to lower or higher tidal volume delivery in a small population of infants with RDS managed with volume guarantee ventilation.

- It is possible that both tidal volume ranges selected for study are near the functional residual capacity and that larger differences might be necessary to show a change. (Figure 7) 